\title{
Dynamical Evolution of Ecliptic Comets
}

\author{
Martin Duncan \\ Queen's University
}

\author{
Harold Levison and Luke Dones \\ Southwest Research Institute
}

\begin{abstract}
Ecliptic comets are those with $\mathrm{T}>2$, where $\mathrm{T}$ is the Tisserand parameter with respect to Jupiter. In this chapter, we review the enormous progress that has been made in our understanding of the dynamical evolution of these bodies. We begin by reviewing the evidence that Jupiter-family comets (JFCs; those with $2<\mathrm{T}<3$ ) form a dynamically distinct class of comets that originate in a flattened disk beyond Neptune. We present a model for the distribution of comets throughout the JFC and Centaur regions that is consistent with current observations, although further observations and numerical simulations in the Centaur region are called for. We then discuss dynamical results (since confirmed by observations) that a significant amount of material that was scattered by Neptune during the early stages of planet formation could persist today in the form of a "scattered disk" of bodies with highly eccentric orbits beyond Neptune. We describe the dynamical mechanisms believed responsible for the longevity of the surviving bodies and argue that if objects in the Kuiper belt and scattered disk have similar size distributions, then the scattered disk is likely to be the primary source of JFCs and Centaurs. Finally, we describe the importance of understanding the ecliptic comet population for the purposes of determining impact rates on the satellites of the giant planets and of age determinations of the satellite surfaces. We present tables of impact rates based on the best currently available analyses. Further refinements of these rates and age determinations await better observations of the Centaur population (including its size distribution), as well as a better understanding of the formation and early dynamical evolution of the outer solar system.
\end{abstract}

\section{INTRODUCTION}

The dynamical and physical lifetimes of most observed comets are short compared to the age of the solar system. Thus, comets must be coming from some reservoir or reservoirs that slowly allow comets to leak out to regions where they can be detected. Although these reservoir(s) must be stable enough to retain a significant number of objects for billions of years, there must also be some currently active mechanisms that transport objects into the regions where they are more easily detected.

It is currently believed that there are three main sources of the known comets: the Oort cloud, the Kuiper belt, and the scattered comet disk. The first of these is discussed in Dones et al. (2004). Here we discuss the Kuiper belt and scattered disk with regard to their role as the source of ecliptic comets. The structure of these cometary reservoirs are discussed in more detail in Morbidelli and Brown (2004).

This chapter is organized as follows. In the next section we briefly review comet taxonomy and terminology. In section 3 we review numerical integrations of the orbits of observed Jupiter-family comets, and in section 4 we summarize results of simulations of the transport of comets from the transneptunian region into the region typically inhabited by JFCs. Section 5 reviews the dynamical properties of the long-lived tail of the distribution of Neptune-scattered comets (the "scattered disk"). In section 6 , the impact rates of ecliptic comets on planets and their moons are discussed, while section 7 contains a brief summary of the state of understanding of the origin of JFCs and the dynamical evolution of ecliptic comets.

\section{COMETARY TAXONOMY}

Historically, cometary taxonomy was based on orbital period, with comets of orbital period shorter than $200 \mathrm{yr}$ being termed "short-period comets" and those with periods less than $20 \mathrm{yr}$ being further subdivided into the class called "Jupiter-family" comets. However, numerical integrations such as those described below show that under such a scheme a given short-period comet typically shifts in and out of the "Jupiter-family class" many times during its dynamical evolution. Carusi and Valsecchi (1987) first suggested that since the Tisserand parameter does not vary substantially during a typical comet's lifetime, a taxonomy based on this parameter might be more appropriate than one based on orbital period.

Recall that the Tisserand parameter with respect to Jupiter, $\mathrm{T}$, is defined as

$$
\mathrm{T}=\mathrm{a}_{\mathrm{J}} / \mathrm{a}+2 \sqrt{\left(1-\mathrm{e}^{2}\right) \mathrm{a} / \mathrm{a}_{\mathrm{J}}} \cos (\mathrm{i})
$$

where $a_{J}$ is Jupiter's semimajor axis, and a, e, and i refer to an object's semimajor axis, eccentricity, and inclination 
respectively. It is an approximation to the Jacobi constant, which is an integral of the motion in the circular restricted three-body problem. It is also a measure of the relative velocity between a comet and Jupiter during close encounters, $\mathrm{v}_{\text {rel }}=\mathrm{v}_{\mathrm{c}} \sqrt{3-\mathrm{T}}$, where $\mathrm{v}_{\mathrm{c}}$ is Jupiter's velocity about the Sun. Objects with $\mathrm{T}$ close to, but smaller than, 3 have very slow, and thus very strong, encounters with Jupiter. Objects with $\mathrm{T}>3$ cannot cross Jupiter's orbit in the circular restricted case, being confined to orbits either totally interior or totally exterior to Jupiter.

For this chapter, we will adopt a taxonomic scheme based on that of Levison (1996), in which the most significant division is based on the Tisserand parameter. In this scheme comets with $\mathrm{T}>2$ are designated ecliptic comets because most members have small inclinations. As we shall see below, these objects most likely originate in the Kuiper belt (Edgeworth, 1949; Kuiper, 1951; Fernández, 1980; Duncan et al., 1988) or the scattered disk (Torbett, 1989; Duncan and Levison, 1997). Comets with $\mathrm{T}<2$, which are believed to be mainly comets from the Oort cloud (Oort, 1950; Everhart, 1977) are designated nearly isotropic comets, reflecting their inclination distribution (see Dones et al., 2004). Independent of other classifications, a comet is said to be "visible" if its perihelion distance is less than 2.5 AU.

Ecliptic comets can be further subdivided into three groups. Comets with $2<\mathrm{T}<3$ are mainly on Jupiter-crossing orbits and are dynamically dominated by that planet. We call these Jupiter-family comets (hereafter called JFCs). Comets with $\mathrm{T}>3$ (not Jupiter-crossing) are not considered members of the Jupiter family. A comet that has $\mathrm{T}>3$ and a $>\mathrm{a}_{\mathrm{J}}$ (orbit is exterior to Jupiter) is designated as Chirontype or a Centaur. A comet that has $\mathrm{T}>3$ and $\mathrm{a}<\mathrm{a}_{\mathrm{J}}$ is designated an Encke-type. Note that this combination of $\mathrm{T}$ and a implies that the orbit of this object is entirely interior to Jupiter, i.e., the aphelion distance is less than $\mathrm{a}_{\mathrm{J}}$. However, it may be too severe to use a strict criteria of $\mathrm{T}<3$ since there are a few comets with $\mathrm{T}$ slightly larger than 3 that dynamically belong to the Jupiter family (i.e., they are not decoupled from Jupiter; they suffer frequent long-lasting encounters with the planet). These are the comets sometimes called the "quasi-Hilda" type (see, e.g., Kresák, 1979; Tancredi et al., 1990). This is a very interesting group since these objects experience frequent temporary satellite capture and occasional low-velocity impacts with Jupiter (like Shoemaker-Levy 9) and Jupiter's moons.

\section{DYNAMICS OF OBSERVED JUPITER-FAMILY COMETS}

Historically, researchers were interested in the origin and evolution of the group of observed comets known as "shortperiod" comets, (i.e., those with periods less than $200 \mathrm{yr}$ ). The upper two panels of Fig. 1 show the distributions of semimajor axis a and inclination i for these comets vs. Tisserand parameter T. The dashed lines in both plots represent the boundaries of the Jupiter family according to our
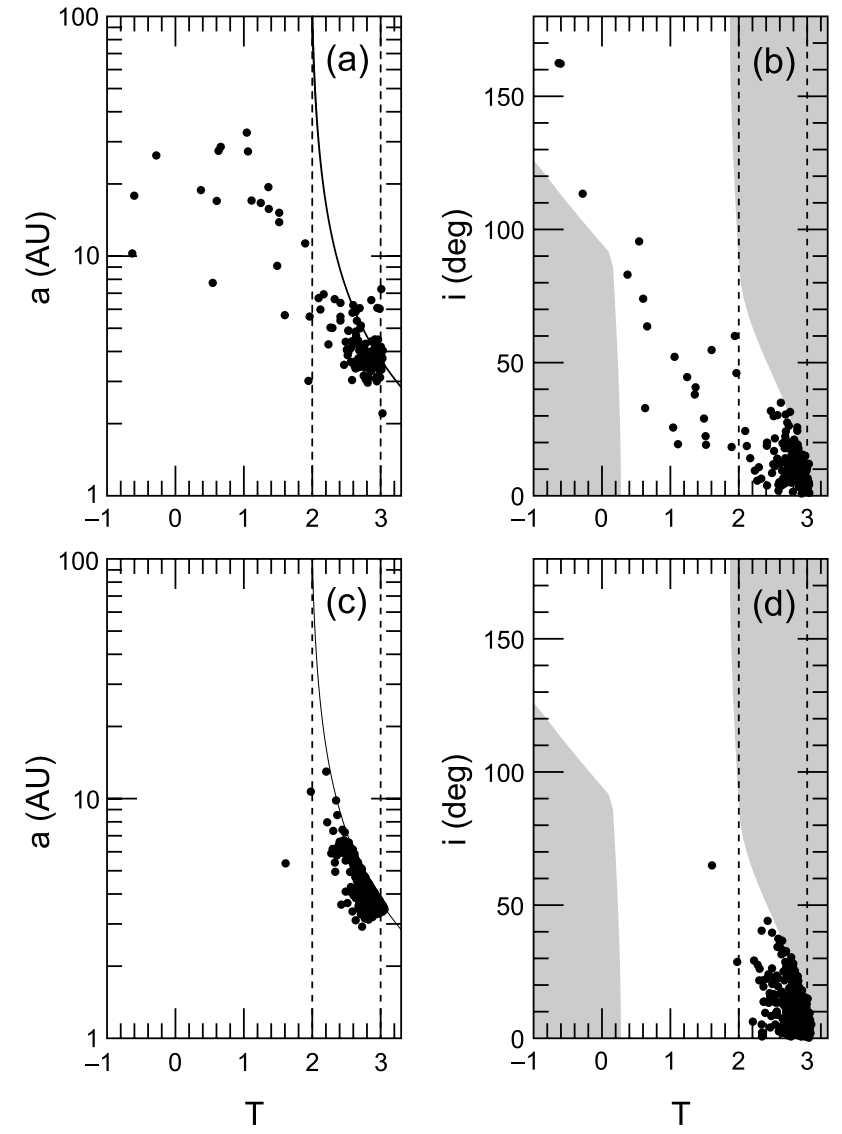

Fig. 1. (a) The semimajor axes, a, of observed comets with periods less than $200 \mathrm{yr}$ as a function of their Tisserand parameter with respect to Jupiter, $\mathrm{T}$. The dashed vertical lines represent the boundaries of the Jupiter family at $\mathrm{T}=2$ and $\mathrm{T}=3$. Objects falling above the solid curve must have perihelia greater than $2.5 \mathrm{AU}$. (b) The inclination, i, of the same set of comets as in (a) as a function of T. Again, the dashed lines represent the boundaries of the Jupiter family. The shaded areas represent regions that are physically unattainable due to the relationship between $\mathrm{i}$ and $\mathrm{T}$ assuming $\mathrm{q} \leq 2.5 \mathrm{AU}$ and $\mathrm{Q} \geq \mathrm{a}_{\mathrm{J}}$. (c) The same as (a) except for simulated comets when they first become "visible," i.e., when their perihelia first drop below 2.5 AU. (d) The same as (b) except for simulated comets when they first become visible. From Levison and Duncan (1997).

taxonomy. The strong clumpings of JFCs at values of T just less than 3 and at low inclinations are important clues to their origins, as we shall describe in section 4.

Pioneering integrations of the dynamical evolution of short-period comets spanning roughly $1000 \mathrm{yr}$ were performed by Carusi et al. (1985) and Carusi and Valsecchi (1987). Further important numerical integrations in the early 1990s include the "COSMO-DICE" project (Nakamura and Yoshikawa, 1992a,b), as well as the work of Tancredi and Rickman (Tancredi and Rickman, 1992; Rickman, 1992; Tancredi, 1994), Lindgren (1992), and Emet'yanenko (1992). 
A comprehensive set of long-term integrations spanning up to $10^{7} \mathrm{yr}$ of the dynamical evolution of short-period comets was performed by Levison and Duncan (1994) (hereafter called $L D 94)$. In view of the chaotic nature of each individual orbit, $L D 94$ integrated 4 orbits per comet (each with slightly different initial orbital elements) for the 160 shortperiod comets known in 1991. The orbits of the Sun, planets (except Mercury and Pluto), and comets were integrated forward and backward in time for $10^{7} \mathrm{yr}$. A comet was followed until it either became unbound from the Sun and reached a distance of $150 \mathrm{AU}$ or became a Sun-grazer with pericenter less than 2 solar radii. Although the chaotic behavior of individual orbits precluded an accurate determination of the long-term fate of any individual comet, it was appropriate to extract statistical information from this sample that should resemble the evolution of the real ensemble of comets.

LD94 found that the Tisserand parameter, $\mathrm{T}$, does not vary substantially for most observed short-period comets: Less than $8 \%$ of comets moved in or out of the JFC class during the integration, and most of those that changed tended to remain near the Tisserand dividing line throughout. Thus, the JFCs were found to be a dynamically distinct class within the short-period comets.

The currently observed perihelion distance distribution of comets is strongly peaked toward small values. This is most likely due to a strong observational bias against the discovery of comets with large perihelion distances because they are less active and do not pass close to the Earth. Quinn et al. (1990) define a "visible" comet as one with $\mathrm{q} \leq 2.5 \mathrm{AU}$. If a comet has a q greater than this value then, they argue, it is not likely to become bright enough to be discovered. Indeed, only $14 \%$ of the known short-period comets have $\mathrm{q}$ larger than this value, despite the fact that there is a large region of phase space with $\mathrm{q}>2.5 \mathrm{AU}$ available to objects being scattered by Jupiter. For this review, we adopt this definition of visibility.

It was found that in the forward integration, $92 \%$ of comets were ejected from the solar system, and that $\approx 6 \%$ were destroyed by becoming Sun-grazers. However, LD94 did not differentiate between JFCs and Halley-type comets (HTCs) $(\mathrm{T}<2, \mathrm{a}<40 \mathrm{AU})$ when considering Sun-grazers. A subsequent reexamination of those integrations found that half the objects that became Sun-grazers were Halley type, although HTCs represented only $\sim 10 \%$ of the comets integrated in $L D 94$. A full $30 \%$ of the HTCs became Sun-grazers, while only $3 \%$ of the JFCs shared the same fate in the integrations. Indeed, only $\sim 1 \%$ of the objects that became visible eventually became Sun-grazers.

The median lifetime of all known short-period comets from the current time to ultimate destruction or ejection is approximately $4.5 \times 10^{5} \mathrm{yr}$ : The median lifetime of JFCs is $3.25 \times 10^{5} \mathrm{yr}$. The median number of times that JFCs changed from orbits with $\mathrm{q}<2.5$ AU to $\mathrm{q}>2.5 \mathrm{AU}$ was 10 . A typical comet spent less than $7 \%$ (median value) of its dynamical lifetime with $\mathrm{q}<2.5$ AU. Since objects with perihelia beyond 2.5 AU are difficult to detect, this implies that there are more than 10 times more undetected JFCs than there are visible JFCs. Of those visible now, half will evolve to states with $\mathrm{q}>2.5 \mathrm{AU}$ in roughly $10^{3} \mathrm{yr}$.

The very flattened inclination distribution of JFCs (i.e., their concentration at small inclinations) was found to become more distended as it aged. Since JFCs are a dynamically distinct class, they must have an inclination distribution, when they first become visible, that is even more flattened than that currently observed. Given this evidence that the JFCs originate in a flattened distribution such as the Kuiper belt, we explore the dynamical transport from the transneptunian reservoirs in the next section.

\section{FROM THE TRANSNEPTUNIAN REGION TO JUPITER-FAMILY COMETS}

We turn now to the study of the origin and dynamical evolution of the class of objects that we referred to in section 2 as "ecliptic comets." As noted in section 3, the observed JFCs (which make up the bulk of the active ecliptic comets) have a very flattened inclination distribution with a median inclination of only $11^{\circ}$. In the last 15 years or so, research attempting to explain this inclination distribution has been extremely active. Indeed, attempting to understand these comets stimulated one of the most important discoveries in planetary science in the last half of the twentieth century - the discovery of the Kuiper belt.

Ecliptic comets were originally thought to originate from nearly isotropic comets that had been captured into shortperiod orbits by gravitational encounters with the planets (Newton, 1891; Everhart, 1977; Bailey, 1986). Joss [(1973), see later work by Fernández and Gallardo (1994) and Levison et al. (2001)] argued that this process is too inefficient, and Fernández (1980) suggested that a belt of distant icy planetesimals beyond Neptune could serve as a more efficient source of most of these comets. Duncan et al. (1988) strengthened this argument by performing dynamical simulations that showed that a cometary source beyond Neptune with small inclinations to the ecliptic was far more consistent with the observed orbits of most of these comets than the randomly distributed inclinations of comets in the Oort cloud (see also Quinn et al., 1990). They named this source the Kuiper belt.

The size, extent, and eccentricity of the cometary orbits in this belt were left as open questions in Duncan et al. (1988). Traditionally, this work was used to imply that the source of these comets is a primordial population of low eccentricity, moderately low-inclination objects beyond Neptune (in what we call the Kuiper belt in this review). An alternative interpretation is that this disk was made up of objects on moderately low-inclination, highly eccentric orbits beyond Neptune (in what we call the scattered disk). The details of these interpretations are discussed below. Nevertheless, the first transneptunian object (after Pluto and Charon) was discovered in 1992 (Luu and Jewitt, 1993); 
now more than 800 transneptunian objects are known (for current lists see http://cfa-www.harvard.edu.edu/ps/lists/ TNOs.html and http://cfa-www.harvard.edu.edu/iau/lists/ Centaurs.html).

To date the only comprehensive simulation of the transport of objects from the transneptunian region to the inner solar system is that of Levison and Duncan (1997) (hereafter LD97). They assumed that the source region was a dynamically very cold primordial Kuiper belt and that there were enough objects leaking out of this belt, due to the gravitational effects of the planets (e.g., Levison and Duncan, 1993; Holman and Wisdom, 1993; Nesvorny and Roig, 2000; Kuchner et al., 2002) to supply the JFCs. Although this assumption is limiting in some respects (which we discuss below), we believe that there are properties of the dynamics of these objects that are independent of the details of the source reservoir. Thus, we discuss the results of LD97 in some detail.

LD97 performed numerical orbital integrations of 2200 massless particles as they evolved from Neptune-encountering orbits in the Kuiper belt for times up to a billion years or until they either impacted a massive body or were ejected from the solar system. The initial orbits for these particles were chosen from a previous set of integrations of objects that were initially in low-eccentricity, low-inclination orbits in the Kuiper belt but evolved onto Neptune-crossing orbits on timescales between 1 and 4 G.y. (Duncan et al., 1995). The median inclination of the particles at the time of encountering Neptune was $4^{\circ}$.

$L D 97$ found that as objects evolve inward from the Kuiper belt, they tend to be under the dynamical control of just one planet. That planet will scatter the comets inward and outward in a random walk, typically handing them off to the planet directly interior or exterior to it. Therefore, the comets tend to have eccentricities of about 0.25 between handoffs. However, once they have been scattered into the inner solar system by Jupiter, they can have much larger eccentricities as they evolve outward.

Plate 7 shows the evolution of a typical particle in the perihelion distance $(\mathrm{q})$-aphelion distance $(\mathrm{Q})$ plane, as it evolves from the Kuiper belt $(q>30$ AU) to a visible JFC (the most populous of the visible ecliptic comets). The positions are joined by blue lines until the particle first became "visible" $(q<2.5 \mathrm{AU})$ and are linked in red thereafter. Initially, the particle spent considerable time with perihelion near the orbit of Neptune (30 AU) and aphelion well beyond the planetary system. However, once its perihelion dropped to Uranus' location, this particle, which was chosen at random from LD97's integrations, clearly shows the handoff behavior described above. It evolved at relatively small eccentricity to visibility (cf. the lines of constant eccentricity e $=0.2$ and 0.3 on Plate 7 ) and it spent considerable time with perihelion or aphelion near the semimajor axis of one of the three outer planets. Its postvisibility phase is reasonably typical of JFCs, with much larger eccentricities than the previsibility comets and perihelion distances near Jupiter or Saturn.
Plate 8 shows the distribution of the ecliptic comets derived from the simulations of $L D 97$, assuming that the rate of objects leaving the Kuiper belt has remained approximately constant over the last $\sim 10^{8} \mathrm{yr}$ and that there was no huge influx at early epochs. The figure is a contour plot of the fraction of comets per square AU in perihelion-aphelion (q-Q) space. The figure was generated by binning the $\mathrm{q}-\mathrm{Q}$ values of all the comets at all output points in the integrations, which occurred once every $10^{4} \mathrm{yr}$ before a comet became visible and once every $1000 \mathrm{yr}$ after it become visible. The resulting matrix was then normalized so that the total number is 1 . Note that we are not plotting, say, the relative number of comets per square $\mathrm{AU}$ at a given distance from the Sun, but rather presenting the more abstract density contours on a grid with pericentric distance $\mathrm{q}$ on one axis and apocentric distance $\mathrm{Q}$ on the other. Also shown in Plate 8 are curves of constant eccentricity and semimajor axis.

There are two well-defined regions in Plate 8. Beyond approximately $\mathrm{Q}=7 \mathrm{AU}$, there is a ridge of high density extending diagonally from the upper right to the center of the plot, near $\mathrm{e} \approx 0.25$. The peak of this ridge is near $\mathrm{e}=0.2$ at large $\mathrm{Q}$ and tends to increase to $\mathrm{e} \approx 0.3$ near $\mathrm{Q} \sim 7 \mathrm{AU}$. As discussed in $L D 97$, the eccentricities of objects that are between the planets and not yet under Jupiter's control are expected to be about 0.25 due to the constraints of the Tisserand parameter. The peak density in this ridge drops by almost 2 orders of magnitude as it moves inward, having a minimum where the semimajor axes of the comets are the same as Jupiter's. This population extends inward of Jupiter's orbit and terminates near the $2: 1$ mean-motion resonance with Jupiter. Indeed, we find that objects can be forced onto nearly circular orbits with semimajor axes as small as $\sim 4$ AU. This inner edge is coincident with the inner edge of Jupiter's "crossing zone" at 3.88 AU. Gladman and Duncan (1990) defined Jupiter's "crossing zone" as the region in which objects on initially circular orbits can become Jupiter-crossing. Since this process is time reversible, it is not then surprising that Jupiter can drive comets into nearly circular orbits in this region.

Inside of $\mathrm{Q} \approx 7 \mathrm{AU}$ the character of the distribution is quite different. Here there is a ridge of high density extending vertically in the figure at $\mathrm{Q} \sim 5-6 \mathrm{AU}$ that extends over a wide range of perihelion distances. Objects in this region are the JFCs. This characteristic of a very narrow distribution in $\mathrm{Q}$ is seen in the real JFCs and is again a result of the narrow range in $\mathrm{T}$. Taken together, this distribution of comets produces a surface distribution shown as the dashed curve in Fig. 2.

The median dynamical lifetime of the ecliptic comets was found to be $4.5 \times 10^{7} \mathrm{yr}$. (This is the time from the first encounter with Neptune to ejection from the solar system, placement in the Oort cloud, which they took to include comets with semimajor axes $>1000$ AU, or impact with the Sun or a planet). $L D 97$ found that about $30 \%$ of the objects in the integrations became visible comets $(q<2.5 \mathrm{AU})$. Of those that became visible, $99.7 \%$ were JFCs at the time of first visibility. 


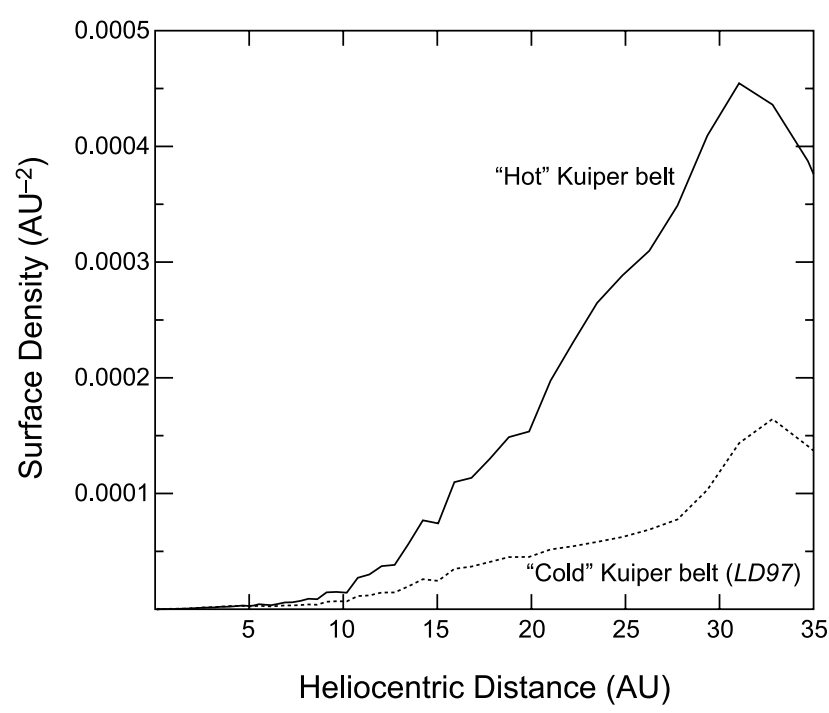

Fig. 2. The predicted surface density of Centaurs and JFCs as a function of heliocentric distance. The dashed curve represents objects initially on low-inclination Kuiper belt orbits in the integrations of $L D 97$. The solid curve is from a small number of objects with initial inclinations of roughly $25^{\circ}$.

In order to compare the results of the simulations to the distribution of real comets, Figs. 1c and 1d show the orbital element distribution of all visible comets in the simulations of $L D 97$ when they first became visible. When making a comparison between the real and simulated JFCs in Fig. 1 it is important to note that there are about five times as many points in the simulated population as in the observed population. Thus, outlying data points in the simulated population are less significant than they may otherwise seem. The distribution of comets in the simulation matches that for the real Jupiter-family remarkably well. Therefore, the simulations show that the Kuiper belt is an excellent potential source for the JFCs. However, the Kuiper belt does not produce very many HTCs (at least initially, see below). This result is consistent with the results of earlier work (Quinn et al., 1990) that the Kuiper belt is the main source of JFCs, whereas another reservoir (possibly the inner Oort cloud) is the most likely source for most of the HTCs (see Dones et al., 2004).

An interesting aspect of the distributions shown in Fig. 1 is the very narrow range in T that JFCs occupy. Although we define a JFC as one with $2<\mathrm{T}<3$, the median $\mathrm{T}$ for the family is 2.8 . The narrow range in $\mathrm{T}$ is related to small inclinations to the ecliptic seen for these comets: An object on a Jupiter-crossing orbit with $\mathrm{T}>2.8$ and $\mathrm{q}<2.5 \mathrm{AU}$ must have an inclination less than $\sim 26^{\circ}$.

The inclination distribution of JFCs when they first become visible is more concentrated to small inclinations than the observed population and was found to become more distended as it aged. Indeed, this model predicts an inclina- tion distribution more extended than the observations unless fading due to physical evolution is included. From this $L D 97$ estimated that the physical lifetime of JFCs is between 3000 and 25,000 yr. The most likely value is $12,000 \mathrm{yr}$.

With this estimate of the physical lifetime, $L D 97$ were able to calibrate the total number of ecliptic comets by using the number of observed JFCs. They thereby estimated that there are roughly a million ecliptic comets with semimajor axes less than $30 \mathrm{AU}$. This estimate refers to objects that would produce active comets with total absolute magnitudes brighter then 9 if they came within $2.5 \mathrm{AU}$ of the Sun. We discuss the conversion from limits based on absolute magnitudes to those based on physical radii in section 5 .

In the simulations of $L D 97$, when the ecliptic comets first became visible they were almost entirely members of the Jupiter family. Although a small fraction of these comets switched to visible HTCs, the orbital element distribution of LD97's simulated HTCs is not consistent with the observed distribution. In particular, the semimajor axes of the simulated comets are generally too small. Since $L D 97$ found that it takes at least $10^{5} \mathrm{yr}$ and usually over $10^{6} \mathrm{yr}$ to become a visible HTC after the comet first becomes visible, most of these comets have likely become extinct since this is longer then the typical physical lifetime estimated above. Thus, although the Kuiper belt can be the source of at least some of the HTCs, initially low-inclination bodies encountering Neptune are unlikely to provide a significant number of them.

Finally, we consider the Encke-type comets, which are low-inclination comets totally interior to Jupiter's orbit (see section 2). Comet 2P/Encke is the only active member of this population. Although 107P/Wilson-Harrington has a T consistent with this class, it is probably not a comet (Bottke et al., 2002). However, there are several kilometer-sized asteroids known to be in similar orbits (Asher et al., 1993). These small "asteroids" could be extinct comets. Numerical integrations of its orbit show that 2P/Encke will hit the Sun in only $10^{5}-10^{6} \mathrm{yr}$ (Levison and Duncan, 1994) due to its close association with secular resonances (Valsecchi et al., 1995).

The LD97 integrations did not produce any comets similar to $2 \mathrm{P} /$ Encke, but included neither the effects of the terrestrial planets nor nongravitational effects. Some of these effects were considered by Steel and Asher (1996), Harris and Bailey (1998), and Asher et al. (2001). Fernández et al. (2002) integrated the orbits of a sample of real JFCs with the terrestrial planets and nongravitational forces included. They found that they could produce objects on Encke-like orbits, but only when strong nongravitational forces (as strong as or greater than those that are estimated to be currently acting on Encke) are included.

\section{DYNAMICS OF THE SCATTERED DISK}

Perhaps the most interesting result of the $L D 97$ simulations was that about $5 \%$ of the particles survived the length of the integration $\left(10^{9} \mathrm{yr}\right)$. All the survivors had semimajor 


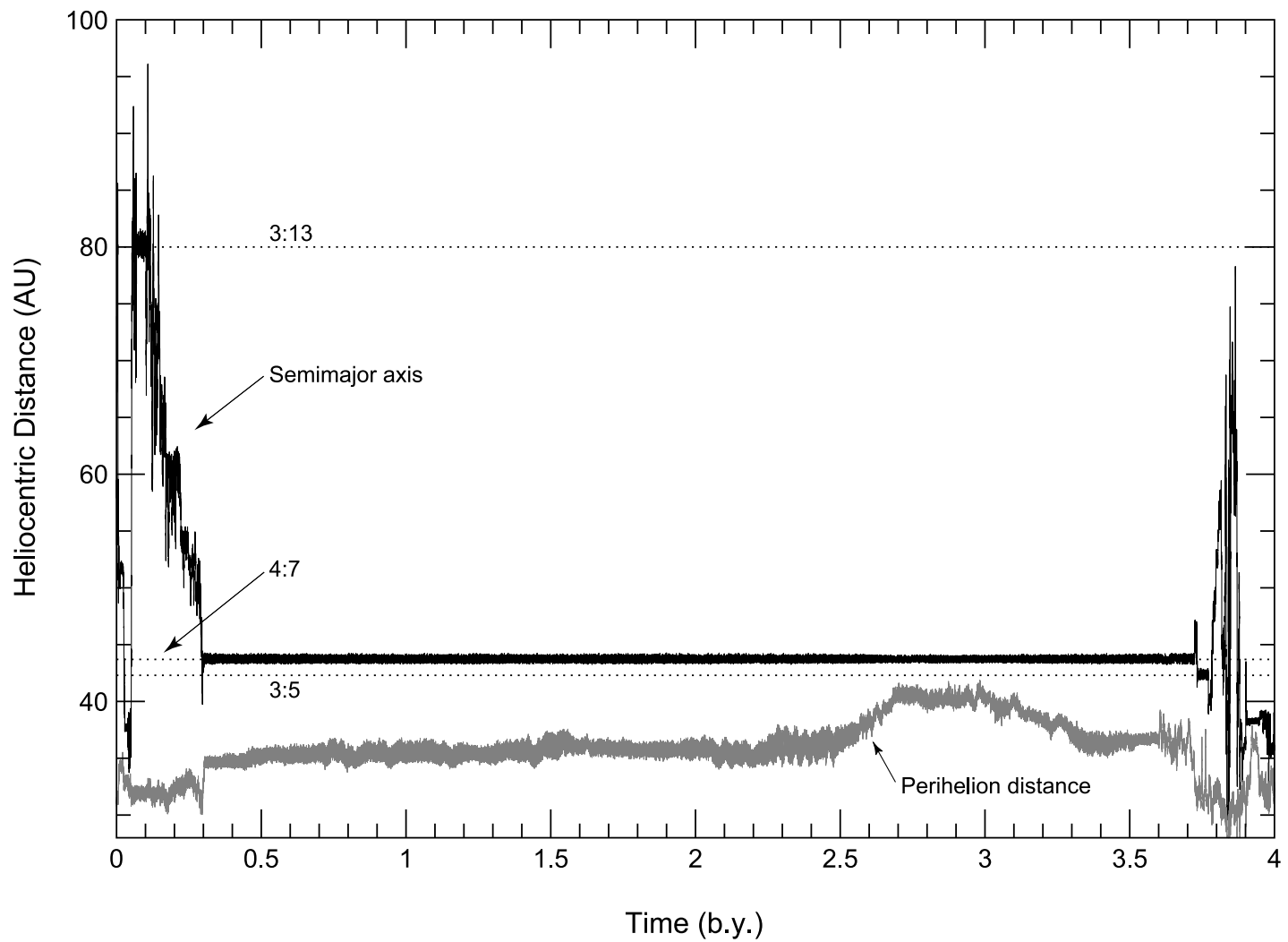

Fig. 3. The temporal behavior of a long-lived member of the scattered disk. The black curve shows the behavior of the comet's semimajor axis. The gray curve shows the perihelion distance. The three dotted curves show the location of the 3:13, 4:7, and 3:5 mean-motion resonances with Neptune. From Duncan and Levison (1997).

axes outside the orbit of Neptune. This result implied that there may be a significant population of objects with highly eccentric orbits in an extended disk beyond the orbit of Neptune - a "scattered" comet disk. Although, the idea of the existence of a scattered comet disk dates to Fernández and Ip (1983) and Torbett (1989), it was the modern work of LD97 and the followup paper, Duncan and Levison (1997) (hereafter DL97), that demonstrated that the scattered disk should exist. DL97 extended the LD97 integrations to $4 \times 10^{9} \mathrm{yr}$. It was found that $1 \%$ of the particles remained in orbits beyond Neptune after 4 b.y. So, if at early times, there was a significant amount of material from the region between Uranus and Neptune or the inner Kuiper belt that evolved onto Neptune-crossing orbits, then there could be a significant amount of this material remaining today. [The first scattered disk object was discovered by Luu et al. (1997) shortly after this prediction.]

DL97 found that some of the long-lived objects were scattered to very long-period orbits where encounters with Neptune became infrequent. However, at any given time, the majority of them were interior to 100 AU. Their longevity is due in large part to their being temporarily trapped in or near mean-motion resonances with Neptune. The "stickiness" of the mean-motion resonances, first mentioned by Holman and Wisdom (1993), leads to an overall distribution of semimajor axes for the particles that is peaked near the locations of many of the mean-motion resonances with Neptune. Occasionally, the longevity is enhanced by the presence of the Kozai resonance (Kozai, 1962).

In all long-lived cases, particles had their perihelion distances increased so that close encounters with Neptune no longer occurred. Frequently, these increases in perihelion distance were associated with trapping in a mean-motion resonance, although in many cases it has not yet been possible to identify the exact process that was involved. On occasion, the perihelion distance can become large, but $81 \%$ of scattered disk objects in the simulations have perihelia between 32 and $36 \mathrm{AU}$.

Figure 3 shows the dynamical behavior of a typical longlived particle. This object initially underwent a random walk in semimajor axis due to encounters with Neptune. At about $7 \times 10^{7} \mathrm{yr}$ it was temporarily trapped in Neptune's 3:13 mean-motion resonance for about $5 \times 10^{7} \mathrm{yr}$. It then performed a random walk in semimajor axis until about $3 \times$ $10^{8} \mathrm{yr}$, when it was trapped in the 4:7 mean-motion resonance, where it remained for $3.4 \times 10^{9} \mathrm{yr}$. Notice the increase in the perihelion distance near the time of capture. While trapped in this resonance, the particle's eccentricity 
became as small as 0.04. After leaving the 4:7, it was trapped temporarily in Neptune's 3:5 mean-motion resonance for $\sim 5 \times 10^{8} \mathrm{yr}$ and then went through a random walk in semimajor axis for the remainder of the simulation.

DL97 estimate the number of scattered disk objects that their model would require if it was the sole source of the JFCs. They first computed the simulated distribution of comets throughout the solar system at the current epoch (assuming the disk was created $4 \times 10^{9} \mathrm{yr}$ ago, with the distribution averaged over the last $10^{9} \mathrm{yr}$ for better statistical accuracy). They found that the ratio of scattered disk objects to visible JFCs (those with a perihelion distance $<2.5 \mathrm{AU}$ ) is $1.3 \times 10^{6}$. Since there are currently estimated to be 500 visible JFCs (LD97; see also Fernández et al., 1999), there are presently $\sim 6 \times 10^{8}$ comets in the scattered disk if this model is the sole source of the JFCs. Figure 4 shows the spatial distribution for this model.

The above estimate is not particularly physically meaningful because it refers to comets with absolute magnitudes, $\mathrm{H}_{\mathrm{T}}$, brighter than 9 when they are visible. A more interesting measure is the number of comets greater than some diameter, typically set to $1 \mathrm{~km}$. Unfortunately, this is a non-

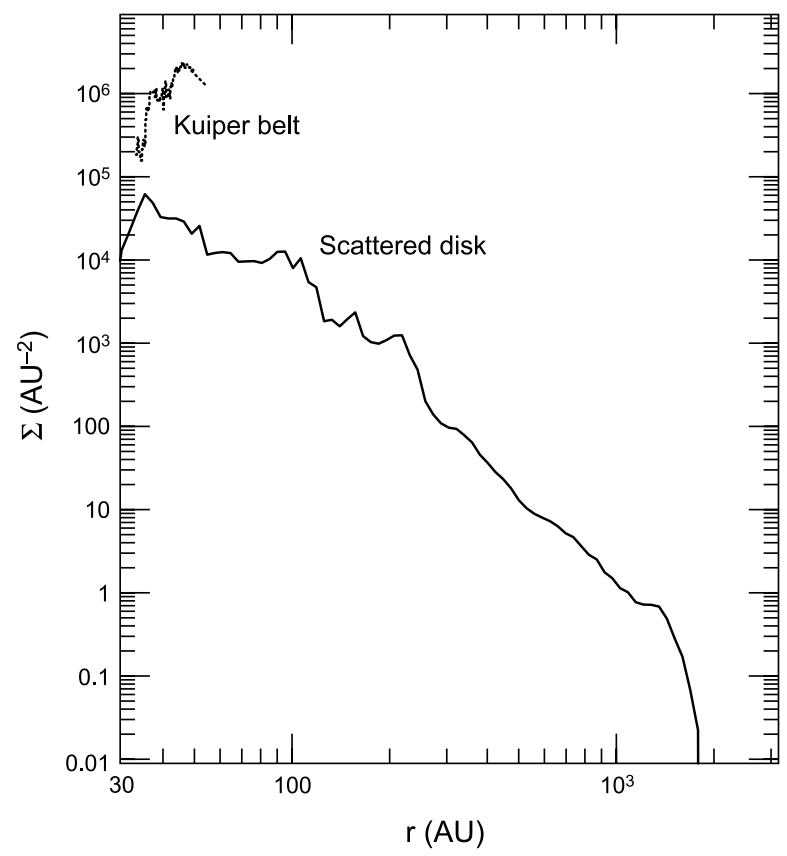

Fig. 4. The surface density of comets beyond Neptune for two different models of the source of JFCs. The dotted curve is the required surface density assuming that a dynamically cold Kuiper belt is the current source (Levison and Duncan, 1997). There are $7 \times 10^{9}$ comets with $\mathrm{H}_{\mathrm{T}}<9$ in this distribution between 30 and $50 \mathrm{AU}$. This curve ends at $50 \mathrm{AU}$ because the models are unconstrained beyond this point and not because it is believed that there are no comets there. The solid curve is the model of Duncan and Levison (1997) assuming the scattered disk produced by their integrations is the sole source of the JFCs. There are estimated to be $6 \times 10^{8}$ comets with $\mathrm{H}_{\mathrm{T}}<9$ currently in this distribution (see text). From Duncan and Levison (1997). trivial conversion, since there is no good correlation between the absolute magnitude of a comet (which is based on a comet's activity) and its size (for discussions, see, e.g., Levison et al., 2001; Zahnle et al., 1998). Examples of possible values for the number of comets in the scattered disk with diameters, $\mathrm{D},>1 \mathrm{~km}$ range from $\sim 5 \times 10^{8}$ [following the calibration of Bailey et al. (1994)], through $\sim 2 \times$ $10^{9}$ [following the calibration of Weissman (1990)], to $\sim 5 \times$ $10^{9}$ (following Levison et al.'s (2001) estimate, based on Kary and Dones (1996)].

If the scattered disk is the source of the Centaurs and JFCs, then the estimate of its population has implications for planet formation. Since we employ the calibration of Weissman (1990) for this discussion, we note that there are significant uncertainties in these numbers. It was shown above that $\sim 1 \%$ of the objects in the scattered disk remain after $4 \times 10^{9} \mathrm{yr}$ in the simulations of $D L 97$, and that $\sim 2 \times 10^{9}$ comets with $\mathrm{D}>1 \mathrm{~km}$ are currently required to supply all the JFCs with the adopted calibration of Weissman (1990). Thus, a scattered comet disk requires an initial population of only $2 \times 10^{11}$ comets [or $\sim 1 \mathrm{M}_{\oplus}$ (Weissman, 1990)] on Neptune-encountering orbits. Since planet formation is unlikely to have been $100 \%$ efficient, the original disk could have resulted from the scattering of even a small fraction of the tens of Earth masses of cometary material that must have populated the outer solar system in order to have formed Uranus and Neptune. Thus, a disk that supplies the current JFCs and Centaurs appears to have properties consistent with that expected from our current understanding of planet formation.

The first scattered disk object discovered was $1996 \mathrm{TL}_{66}$, found in October 1996 by Jane Luu and colleagues (Luu et al., 1997). Current observations indicate that it has a semimajor axis of $85 \mathrm{AU}$, a perihelion of $35 \mathrm{AU}$, and an inclination of $24^{\circ}$. Dozens of other objects are currently known (see http://cfa-www.harvard.edu/iau/lists/Centaurs.html for a complete list.) The total number in 100-km-sized scattered disk objects is estimated to be between 20,000 and 50,000 (Trujillo et al., 2000), comparable to the number of similar-sized Kuiper belt objects interior to 50 AU. The number in comet-sized $(1-10-\mathrm{km})$ bodies remains to be observationally measured. However, using the size distribution of Weissman and Levison (1997) and Trujillo et al.'s (2000) number, we find that we should expect between $2 \times 10^{9}$ and $6 \times 10^{9}$ objects with $\mathrm{D}>1 \mathrm{~km}$. This number is consistent with our estimates above for a scattered disk source of JFCs.

Indeed, we believe that it can now be argued that the scattered disk is the primary source of the Centaurs and JFCs. If the Kuiper belt were the source, we would expect that the number of objects in the Kuiper belt would be more than an order of magnitude larger than the number of objects in the scattered disk because the average dynamical lifetime of scattered disk objects $\left[2 \times 10^{8} \mathrm{yr}\right.$ (Levison et al., 2001)] is much shorter than that of a Kuiper belt object (Duncan et al., 1995; Kuchner et al., 2002). However, we have just noted that Trujillo et al. (2000) found that the two populations of $100-\mathrm{km}$ objects are comparable. Assuming 
that objects in the scattered disk and the Kuiper belt have similar size distributions, the fact that most scattered disk objects are on less stable orbits (see Emet'yanenko et al., 2003) and become planet-crossing at a greater rate implies that the scattered disk is the primary source of the JFCs.

This conclusion is also supported by the recent work of Morbidelli et al. (2004), who performed a detailed comparison between the two different dynamical models for the scattered disk. They found that the orbital element distribution of observed scattered disk objects is not consistent with a scattered disk that is in dynamical equilibrium with the Kuiper belt. Such a model predicts many more scattered disk objects with semimajor axes between 50 and $60 \mathrm{AU}$ (compared to objects with larger semimajor axes) than are actually observed. However, a model invoking an ancient scattered disk, previously much more massive, is in good agreement with the observed scattered disk. Again, we can conclude that the scattered disk is old and is the most likely source of the Centaurs and JFCs.

Very recently, strong evidence has emerged for the existence of an "extended scattered disk" (Gladman et al., 2002). This disk is comprised of bodies such as $2000 \mathrm{CR}_{105}$ that have very large semimajor axes and perihelia outside 40 AU. These objects cannot have been placed on such orbits by strong gravitational scattering off any of the giant planets in their current orbits. The mass in this population is extremely uncertain due to the difficulty in finding such distant objects in the first place and due to the difficulty of determining their orbits without frequent and well-timed recovery observations. Their cosmogonic implications are significant and are discussed in Morbidelli and Brown (2004).

\section{PLANETARY IMPACT RATES FROM ECLIPTIC COMETS}

An important aspect of the ecliptic comet population is that it is the primary source of impactors on the satellites of the giant planets (Zahnle et al., 1998). Thus, if it is possible to determine the total number and orbital distribution of these objects, it should be possible to estimate the ages of the satellite surfaces. Given the importance of this topic, we dedicate this section to this issue.

As described in section 4, the best set of integrations for this purpose remains those presented in LD97. However, before we proceed with a discussion of their results with regard to impact rates, we first need to ask whether the LD97 model is a good representation of the Centaur (i.e., impacting) population. We do this by comparing the orbital element distribution of LD97's synthetic Centaurs to real objects. Unfortunately, the data suffer from significant observational biases in semimajor axis (a), inclination (i), and perihelion distance (q). Thus, to compare the $L D 97$ model to observations, we first run the model through a synthetic survey simulator. Here we use one developed by Morbidelli et al. (2004) for the transneptunian population. This simulator, in turn, was based on the work of Brown (2001).

Our basic procedure is as follows. We generate a set of synthetic Centaurs from the integration of $L D 97$, restrict- ing ourselves to objects with $5.2<\mathrm{a}<30$ AU. For each of these we randomly choose an absolute magnitude, the distribution of which is consistent with what is observed in the Kuiper belt $\left[\mathrm{N}(<\mathrm{H}) \propto 10^{\alpha \mathrm{H}}\right.$, where $\mathrm{H}$ is the absolute magnitude, $\mathrm{N}$ is the total number, and $\alpha=0.7$ (Gladman et al., 2001; Trujillo et al., 2001). We run these objects through the survey simulator, which calculates the probability that each object would have been discovered by the surveys that have thus far discovered real Centaurs. From this, we can generate an estimate of what the model predicts for the orbital element distribution of the known Centaurs. This result is represented by the dark solid curves in Fig. 5. The dotted curves show the distribution of real, multiopposition, Centaurs. The distributions in a, i, and $\mathrm{q}$ are very similar. Thus, we can conclude that the models of $L D 97$ do indeed match the distribution of the currently observed Centaurs.

However, this result is a necessary but not sufficient condition, because the observations may not sample enough of the Centaurs to be a significant constraint on the overall distribution. For example, $L D 97$ studied the evolution of a small number of objects leaving the Kuiper belt at inclinations of $\sim 25^{\circ}$. The solid curve in Fig. 2 shows the surface density distribution of this model compared to the standard LD97 model shown by the dotted curve. Note that there is roughly a factor of three difference between the curves in the outer solar system and a factor of 2 at Saturn. However, they agree at Jupiter.

We have run this "hot" model of the Centaurs through our synthetic survey simulator and the predicted orbital element distribution of discovered objects is shown as the blue curves in Fig. 5. There is reasonably good agreement between this model, the original model, and the observations. The only exception is in the inclinations where objects in this model have slightly (but not significantly) larger inclinations. However, we conclude that the observations cannot yet distinguish between these two reasonable models of the Centaurs. This fact should be considered when interpreting the impacts rates we present below.

Having said this, we return to the issue of impact rates. Included in the analysis presented in LD97 was a crude estimate of the impact rates on the planets from ecliptic comets. Subsequently, Levison et al. (2000) have used the results of $L D 97$ to present a more precise and detailed analysis of the impact rates and characteristics of the impacting bodies. Levison et al. (2000) used three different methods to compute the impact rates on the giant planets (see section 2 of that paper for details). The main results of these calculations are given as the last three columns in their Table 1, which is reproduced here (see Table 1). For this discussion, we note that the authors recommend using the last column (Öpik II) as the standard impact rate: The variation in the entries for each planet should be viewed as a measure of the uncertainty in the rate given in the last column.

The impact rates were calculated assuming that the rate at which comets evolve from their source region to the ecliptic comet population has been constant. This assumption is clearly not correct, although the authors believe that it does 

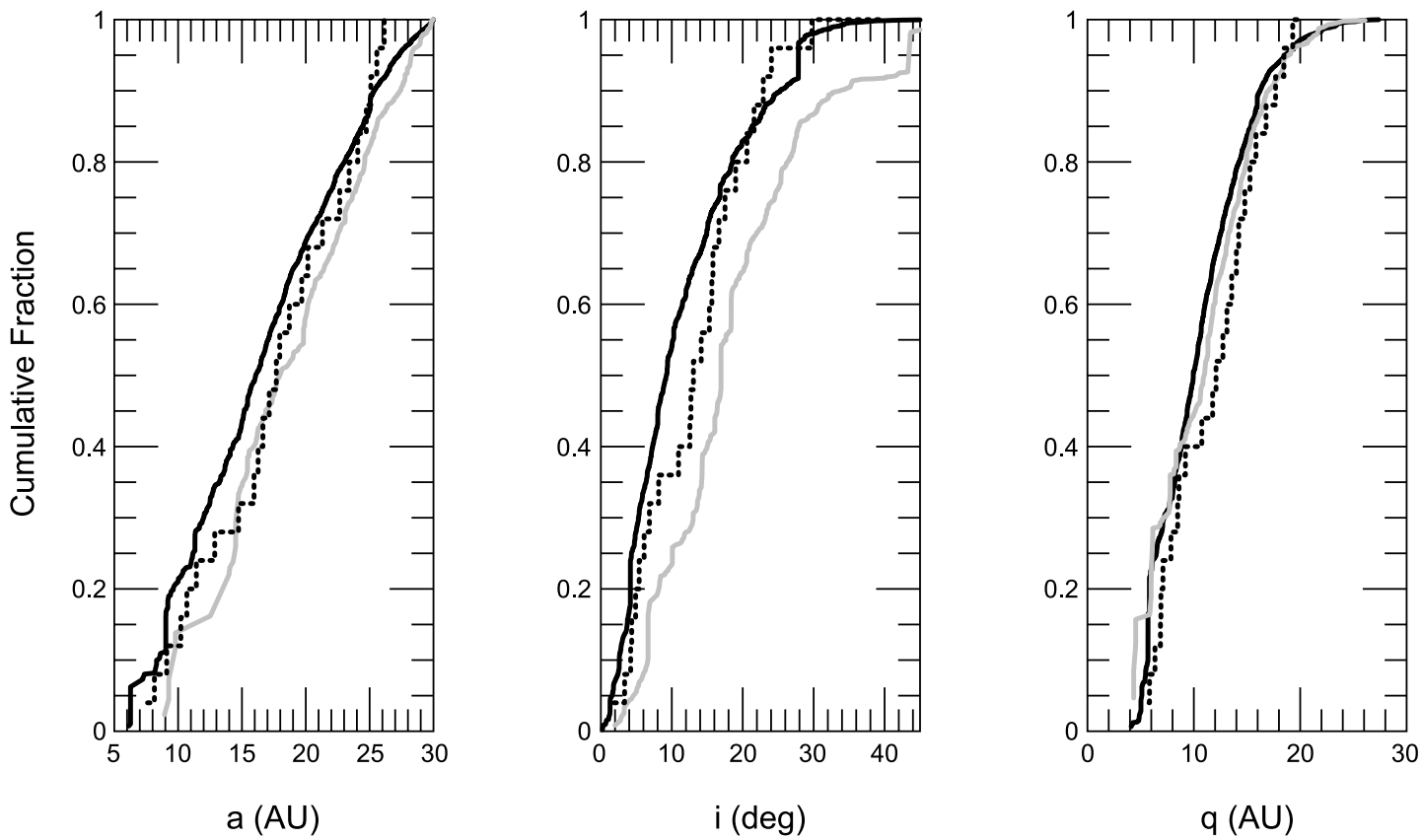

Fig. 5. The orbital element distribution of the Centaurs. The dark solid curves are those derived from the simulations of $L D 97$, while the gray curves are derived from a subset that started with objects leaving the Kuiper belt with inclinations of roughly $25^{\circ}$. Both the solid curves were generated using the synthetic survey simulator of Morbidelli et al. (2004). The dotted curves show the distribution of observed, multiopposition, Centaurs.

TABLE 1. Impact rates of ecliptic comets with $\mathrm{H}_{\mathrm{T}}<9$ on the planets according to Levison et al. (2000).

\begin{tabular}{lccccc}
\hline Planet & $\begin{array}{c}\text { Number of } \\
\text { Impacts } \\
\text { Stage } 1\end{array}$ & $\begin{array}{c}\text { Number of } \\
\text { Impacts } \\
\text { Stage } 2\end{array}$ & $\begin{array}{c}\text { Rate Direct } \\
(\text { comets/yr) }\end{array}$ & $\begin{array}{c}\text { Rate Öpik I } \\
(\text { comets/yr) }\end{array}$ & $\begin{array}{c}\text { Rate Öpik II } \\
(\text { comets/yr) }\end{array}$ \\
\hline Jupiter & 7 & 114 & $6.3 \times 10^{-4}$ & $5.0 \times 10^{-4}$ & $6.5 \times 10^{-4}$ \\
Saturn & 4 & 17 & $1.9 \times 10^{-4}$ & $2.0 \times 10^{-4}$ & $2.7 \times 10^{-4}$ \\
Uranus & 10 & 0 & $3.4 \times 10^{-4}$ & $1.2 \times 10^{-4}$ & $1.6 \times 10^{-4}$ \\
Neptune & 10 & 0 & $3.4 \times 10^{-4}$ & $2.6 \times 10^{-4}$ & $3.5 \times 10^{-4}$ \\
Mercury & - & - & - & $4.8 \times 10^{-9}$ & $6.1 \times 10^{-9}$ \\
Venus & - & - & - & $4.2 \times 10^{-8}$ & $5.4 \times 10^{-8}$ \\
Earth & - & - & - & $6.2 \times 10^{-8}$ & $8.0 \times 10^{-8}$ \\
Mars & - & - & & $1.4 \times 10^{-8}$ & $1.8 \times 10^{-8}$ \\
\hline
\end{tabular}

not have a large effect on the estimates of the current impact rates. However, most likely these impact rates were significantly higher in the distant past. For example, if DL97's model of the scattered disk is correct, the impact rates on the giant planet satellites $2 \times 10^{9} \mathrm{yr}$ ago were roughly a factor of 2 larger than current rates. Any attempt to use the impact rates to estimate the ages of satellites should take this into account.

The impact rates given in Table 1 are calibrated to active JFCs with absolute magnitudes, $\mathrm{H}_{\mathrm{T}}$, brighter than 9. As describe above, a more common and standardized measure of impact rates is to present them scaled to the number of objects with diameters greater than $1 \mathrm{~km}$ striking a planet per year. In order to do this, Levison et al. (2000) find that the values presented in Table 1 should be multiplied by a factor of $\sim 5$, although there are great uncertainties in this number. For example, Bottke et al. (2002) use data from the Spacewatch Near Earth Asteroid survey to estimate that this scale factor should be $1.7 \pm 1.2$ rather than 5 . However, this assumes that $100 \%$ of all JFCs become inactive rather than disintegrate once their active lifetime is over. If two-thirds of the JFC population self-destructed, the values of the scale factor would agree.

Zahnle et al. $(1998,2001,2003)$ computed impact rates on the satellites of Jupiter, Saturn, Uranus, and Neptune, and on Pluto/Charon. Ecliptic comets appear to be the main 
impactors on all these bodies. In these papers, the (relative) spatial distribution of ecliptic comets was taken from the integrations of LD97 and DL97. However, the authors attempted to perform a much more careful analysis of the uncertainties in the total number of comets in the system.

Galileo observations of the galilean satellites indicate a paucity of small craters relative to an extrapolation of the distribution at larger sizes (see Schenk et al., 2003, for a discussion.) The most reasonable interpretation of this result is that there are relatively few subkilometer comets in the JFC population (at least at Jupiter), which may have important implications for the origins of these objects. Recently, Zahnle et al. (2003) argued that this relative paucity must exist all the way out to 30 AU because if not, lifetimes against collisional disruption by ecliptic comets would be $\ll 10^{9}$ yr for a number of small moons around Saturn, Uranus, and Neptune.

However, if subkilometer comets really are underabundant in the outer solar system, this implies that either (1) there are few such bodies in the source regions - the scattered disk and/or (less likely) the Kuiper belt — or that (2) most small comets are destroyed at heliocentric distances greater than $30 \mathrm{AU}$. Both these explanations have problems. The former seems unlikely because the transneptunian population is generally believed to have been collisional in the early days of the solar system, and therefore should have produced many small fragments (Stern, 1995; Kenyon, 2002; Stern and Weissman, 2001). The latter is unlikely because there is no obvious way to destroy the comets by thermal or other effects at such great distances from the Sun. However, the size distribution of comet precursors likely will not be known until transneptunian occultation experiments such as TAOS (Alcock et al., 2003) are discovering kilometer-sized bodies in the region.

\section{SUMMARY}

In the past 15 years there has been enormous progress in our understanding of the dynamical evolution of ecliptic comets, largely due to the ability of researchers to perform orbital integrations of large numbers of comets for timescales on the order of the age of the solar system. The study of the dynamics of comets has helped lead the way to important discoveries about new structures in the solar system, including the Kuiper belt and the scattered disk.

We began this chapter by reviewing the evidence from numerical integrations that observed that JFCs form a dynamically distinct class of comets. Results were discussed that showed that JFCs, upon first becoming visible, have an inclination distribution that is even more flattened than that currently observed. We then reviewed the compelling dynamical evidence that JFCs and Centaurs originate in a flattened disk beyond Neptune.

A model was described for the distribution of comets throughout the JFC and Centaur regions. The integrations that produced the distribution (LD97) assumed that the original orbits of the comets had very low inclinations, which more recent observations suggest is not the case for a large fraction of the transneptunian population. However, we presented evidence in this review that suggested that many of the properties of the distribution (such as the distribution interior to Jupiter) were independent of the detailed structure of the source reservoir. Indeed, we showed that the predicted Centaur orbital distributions in LD97 are consistent with the distribution of the currently observed, multiopposition Centaurs once observational biases are included. We also noted, however, that the study of a small number of objects leaving the Kuiper belt at inclinations of approximately $25^{\circ}$ also produced a Centaur distribution in rough agreement with the observations despite differing in surface density by a factor of 2-3 from the dynamically "colder" model in the outer planetary region. It is clear that further observations, coupled with a better understanding of the formation and early dynamical evolution of the outer solar system, will be required before these uncertainties can be reduced.

We have presented the dynamical results (since confirmed by observation) that a significant amount of material that was scattered by Neptune during the early stages of planet formation could persist today in the form of a "scattered disk" of bodies with highly eccentric orbits beyond Neptune. We described the dynamical mechanisms believed responsible for the longevity of the subset of objects that have survived to the current epoch. The results of the integrations suggest that the dynamical lifetime of scattered disk objects is much less than that of most currently observed Kuiper belt objects. Since the numbers of objects inferred from observations in the two groups are comparable, then if the two groups have similar size distributions, we argue that the scattered disk is likely to be the primary source of JFCs and Centaurs.

Finally, we have described the importance of understanding the ecliptic comet population for the purposes of determining impact rates on the satellites of the giant planets and of age determinations of the satellite surfaces. We have presented tables of impact rates based on the best currently available analyses. Further refinements of these rates and age determinations await better observations of the Centaur population (including its size distribution), as well as a better understanding of the formation and early dynamical evolution of the outer solar system.

\section{REFERENCES}

Alcock C. and 17 colleagues (2003) The Taiwanese-American Occultation Survey. In Proceedings of the First Decadal Review of the Kuiper Belt (J. Davies and L. Barrera, eds.). Special issue of Earth Moon Planets, 92, in press.

Asher D. J., Clube S. V. M., and Steel D. I. (1993) Asteroids in the Taurid complex. Mon. Not. R. Astron. Soc., 264, 93.

Asher D. J., Bailey M. E., and Steel D. I. (2001) The role of nongravitational forces in decoupling orbits from Jupiter. In Collisional Processes in the Solar System (M. Marov and H. Rickman, eds.), pp. 121-130. Kluwer, Dordrecht. 
Bailey M. E. (1986) The near-parabolic flux and the origin of short-period comets. Nature, 324, 350-352.

Bailey M., Clube S., Hahn G., Napier W., and Valsecchi G. (1994) Hazards due to giant comets: Climate and short-term catastrophism. In Hazards Due to Comets and Asteroids (T. Gehrels et al., eds.), p. 479. Univ. of Arizona, Tucson.

Bottke W., Morbidelli A., Jedicke R., Petit J.-M., Levison H., Michel P., and Metcalfe T. (2002) Debiased orbital and absolute magnitude distribution of the near-earth objects. Icarus, 156, 399-433.

Brown M. (2001) The inclination distribution of the Kuiper Belt. Astron. J., 121, 2804-2814.

Carusi A. and Valsecchi G. (1987) Dynamical evolution of shortperiod comets. In Interplanetary Matter (Z. Ceplecha and P. Pecina, eds.), pp. 21-28. Astronicky ustav CSAV, Praha.

Carusi A., Kresák, L., Perozzi E., and Valsecchi G. (1985) LongTerm Evolution of Short-Period Comets. Adam Hilger, Bristol. $272 \mathrm{pp}$.

Dones L., Weissman P. R., Levison H. F., and Duncan M. J. (2004) Oort cloud formation and dynamics. In Comets II (M. C. Festou et al., eds.), this volume. Univ. of Arizona, Tucson.

Duncan M. and Levison H. (1997) A scattered comet disk and the origin of Jupiter family comets. Science, 276, 1670-1672.

Duncan M., Quinn T., and Tremaine S. (1988) The origin of shortperiod comets. Astrophys. J. Lett., 328, L69-L73.

Duncan M., Levison H., and Budd M. (1995) The dynamical structure of the Kuiper Belt. Astron. J., 110, 3073.

Edgeworth K. (1949) The origin and evolution of the Solar System. Mon. Not. R. Astron. Soc., 109, 600-609.

Emeł'yanenko V. (1992) Evolution of orbits of comets having close encounters with Jupiter. I - Analysis of the effect of errors on the initial system of elements. II - Analysis of the effect of Jupiter's oblateness. Astron. Vestnik, 26, 24.

Emeł'yanenko V., Asher D., and Bailey M. (2003) A new class of trans-neptunian objects in high-eccentricity orbits. Mon. Not. R. Astron. Soc., 338, 443-451.

Everhart E. (1977) The evolution of comet orbits as perturbed by Uranus and Neptune. In Comets, Asteroids, Meteors (A. H. Delsemme, ed.), pp. 99-104. Univ. of Toledo, Ohio.

Fernández J. A. (1980) On the existence of a comet belt beyond Neptune. Mon. Not. R. Astron. Soc., 192, 481-491.

Fernández J. A. and Gallardo T. (1994) The transfer of comets from parabolic orbits to short-period orbits: Numerical studies. Astron. Astrophys., 281, 911-922.

Fernández J. A. and Ip W.-H. (1983) On the time evolution of the cometary influx in the region of the terrestrial planets. Icarus, 54, 377-387.

Fernández J. A., Tancredi G., Rickman H., and Licandro J. (1999) Are there many inactive Jupiter-family comets among the nearearth asteroid population? Astron. Astrophys., 352, 327-340.

Fernández J. A., Gallardo T., and Brunini A. (2002) Are there many inactive Jupiter-family comets among the near-earth asteroid population? Icarus, 159, 358-368.

Gladman B. and Duncan M. (1990) On the fates of minor bodies in the outer solar system. Astron. J., 100, 1680-1693.

Gladman B., Kavelaars J. J., Petit J.-M., Morbidelli A., Holman M. J., and Loredo T. (2001) Evidence for an extended scattered disk. Astron. J., 122, 1051-1066.

Gladman B., Holman M. J., Grav T., Kavelaars J. J., Nicholson P., Aksnes K., and Petit J.-M. (2002) Evidence for an extended scattered disk. Icarus, 157, 269-271.

Harris N. W. and Bailey M. (1998) Dynamical evolution of com- etary asteroids. Mon. Not. R. Astron. Soc., 297, 1227-1236.

Holman M. J. and Wisdom J. (1993) Dynamical stability in the outer solar system and the delivery of short period comets. Astron. J., 105, 1987-1999.

Joss P. (1973) On the origin of short-period comets. Astron. Astrophys., 25, 271.

Kary D. and Dones L. (1996) Capture statistics of short-period comets: Implications for comet D/Shoemaker-Levy 9. Icarus, 121, 207-224.

Kenyon S. J. (2002) Planet formation in the outer solar system. Publ. Astron. Soc. Pacific, 114, 265-283.

Kozai Y. (1962) Secular perturbations of asteroids with high inclination and eccentricity. Astron. J., 67, 591.

Kresák L. (1979) Dynamical interrelations among comets and asteroids. In Asteroids (T. Gehrels, ed.), pp 289-309. Univ. of Arizona, Tucson.

Kuchner M. J., Brown M. E., and Holman M. (2002) Long-term dynamics and the orbital inclinations of the classical Kuiper Belt objects. Astron. J., 124, 1221-1230.

Kuiper G. P. (1951) O the origin of the solar system. In Astrophysics: A Topical Symposium (J. A. Hynek, ed.), p. 357. McGraw-Hill, New York.

Levison H. (1996) Comet taxonomy. In Completing the Inventory of the Solar System (T. W. Rettig and J. M. Hahn, eds.), pp. 173-191. Astronomical Society of the Pacific, San Francisco.

Levison H. and Duncan M. (1993) The gravitational sculpting of the Kuiper Belt. Astrophys. J., 406, 35-38.

Levison H. and Duncan M. (1994) The long-term dynamical behavior of short-period comets. Icarus, 108, 18-36.

Levison H. and Duncan M. (1997) From the Kuiper Belt to Jupiter-family comets: The spatial distribution of ecliptic comets. Icarus, 127, 13-32.

Levison H. F., Duncan M. J., Zahnle K., Holman M., and Dones L. (2000) NOTE: Planetary impact rates from ecliptic comets. Icarus, 143, 415-420.

Levison H. F., Dones H., and Duncan M. J. (2001) The origin of Halley-type comets: Probing the inner Oort cloud. Astron. J., 121, 2253-2267.

Lindgren M. (1992) Dynamical timescales in the Jupiter family. In Asteroids, Comets, Meteors 1991 (A. W. Harris and E. Bowell, eds.), pp. 371-374. Lunar and Planetary Institute, Houston.

Luu J. and Jewitt D. (1993) Discovery of the candidate Kuiper Belt object 1992 QB1. Nature, 362, 730-732.

Luu J., Jewitt D., Trujillo C. A., Hergenrother C. W., Che J., and Offutt W. B. (1997) A new dynamical class in the trans-neptunian solar system. Nature, 387, 573.

Morbidelli A. and Brown M. E. (2004) The Kuiper belt and the primordial evolution of the solar system. In Comets II (M. C. Festou et al., eds.), this volume. Univ. of Arizona, Tucson.

Morbidelli A., Emeł'yanenko V., and Levison H. F. (2004) Origin and orbital distribution of the trans-Neptunian scattered disk. Mon. Not. R. Astron. Soc., in press.

Nakamura T. and Yoshikawa M. (1992a) Long-term orbital evolution of short-period comets found in project "Cosmo-Dice". In Asteroids, Comets, Meteors 1991 (A. W. Harris and E. Bowell, eds.), p. 433. Lunar and Planetary Institute, Houston.

Nakamura T. and Yoshikawa M. (1992b) Invisible comets on evolutionary track of short-period comets. Cel. Mech. Dyn. Astron., 54, 261-266.

Nesvorny D. and Roig F. (2000) Mean motion resonances in the 
trans-neptunian region. I. The 2:3 resonance with Neptune. Icarus, 148, 282-300.

Newton H. A. (1891) Capture of comets by planets. Astron. J., $11,73-75$.

Oort J. H. (1950) The structure of the cloud of comets surrounding the Solar System and a hypothesis concerning its origin. Bull. Astron. Inst. Neth., 11, 91-110.

Quinn T. R., Tremaine S., and Duncan M. J. (1990) Planetary perturbations and the origins of short-period comets. Astrophys. J., 355, 667-679.

Rickman H. (1992) Structure and evolution of the Jupiter family. Cel. Mech. Dyn. Astron., 54, 63-69.

Schenk P. M., Chapman C. R., Zahnle K., and Moore J. (2003) Ages and interiors: The cratering record of the galilean satellites. In Jupiter: The Planet, Satellites, and Magnetosphere (F. Bagenal et al., eds.), in press. Cambridge Univ., New York.

Steel D. I. and Asher D. J. (1996) On the origin of Comet Encke. Mon. Not. R. Astron. Soc., 281, 937-944.

Stern S. A. (1995) Collisional time scales in the Kuiper disk and their implications. Astron. J., 110, 856.

Stern S. A. and Weissman P. (2001) Rapid collisional evolution of comets during the formation of the Oort cloud. Nature, 409, 589-591.

Tancredi G. (1994) Physical and dynamical evolution of Jupiter family comets: Simulations based on the observed sample. Planet. Space Sci., 42, 421-433.

Tancredi G. and Rickman H. (1992) The evolution of Jupiter family comets over 2000 years. In Chaos, Resonance and Collective Dynamical Phenomena in the Solar System (S. Ferraz-
Mello, ed.), p. 269. IAU Symposium 152, Kluwer, Dordrecht. Tancredi G., Lindgren M., and Rickman H. (1990) Temporary satellite capture and orbital evolution of Comet P/Helin-RomanCrockett. Astron. Astrophys., 239, 375-380.

Torbett M. (1989) Chaotic motion in a comet disk beyond Neptune - The delivery of short-period comets. Astron. J., 98, $1477-1481$.

Trujillo C. A., Jewitt D. C., and Luu J. X. (2000) Population of the scattered Kuiper Belt. Astrophys. J., 529, 102-106.

Trujillo C. A., Jewitt D. C., and Luu J. X. (2001) Properties of the trans-Neptunian belt: Statistics from the CFHT survey. Astron. J., 122, 457-473.

Valsecchi G. B., Morbidelli A., Gonczi R., Farinella P., Froeschlé Ch., and Froeschlé C. (1995) The dynamics of objects in orbits resembling that of P/Encke. Icarus, 118, 169-180.

Weissman P. R. (1990) The cometary impactor flux at the earth. In Global Catastrophes in Earth History (V. L. Sharpton and P. D. Ward, eds.), pp. 171-180. GSA Special Paper 247.

Weissman P. R. and Levison H. (1997) The population of the trans-neptunian region The Pluto-Charon environment. In Pluto and Charon (S. A. Stern and D. J. Tholen, eds.), p. 559. Univ. of Arizona, Tucson.

Zahnle K., Dones L., and Levison H. F. (1998) Cratering rates on the Galilean satellites. Icarus, 136, 202-222.

Zahnle K., Schenk P., Sobieszczk S., Dones L., and Levison H. F. (2001) Differential cratering of synchronously rotating satellites by ecliptic comets. Icarus, 153, 111-129.

Zahnle K., Schenk P., Levison H., and Dones L. (2003) Cratering rates in the outer solar system. Icarus, 163, 263-289. 